\title{
Discrimination of Dental Pulp Stem Cell Regenerative Heterogeneity by Single Cell Raman Spectroscopy
}

Amr Alraies, $\mathrm{PhD},{ }^{1}$ Elisabetta Canetta, $\mathrm{PhD},{ }^{2}$ Rachel J. Waddington, $\mathrm{PhD},{ }^{1}$ Ryan Moseley, $\mathrm{PhD},{ }^{1 *}$ Alastair J. Sloan, $\mathrm{PhD},{ }^{1}$

\begin{abstract}
${ }^{1}$ Regenerative Biology Group, School of Dentistry, Cardiff Institute of Tissue Engineering and Repair (CITER), College of Biomedical and Life Sciences, Cardiff University, UK. ${ }^{2}$ Faculty of Sports, Health and Applied Science, St Mary's University, London, UK.
\end{abstract}

Amr Alraies. Tel.: +44 (0)2920 745959; Email: AlraiesA@cardiff.ac.uk.

Elisabetta Canetta. Tel: +44 (0)20 8240 4095; Email: Elisabetta.Canetta@ stmarys.ac.uk. Rachel J. Waddington. Tel: +44 (0)29 2251 0647; Email: WaddingtonRJ@ cardiff.ac.uk. Ryan Moseley (Corresponding Author) ${ }^{*}$. Tel: $\quad+44 \quad(0) 292251$ 0649; Email: MoseleyR@ cardiff.ac.uk.

Alastair J. Sloan. Tel.: +44 (0)29 2251 0650; Email: SloanAJ@ cardiff.ac.uk.

Keywords: Dental pulp, stem cells, heterogeneity, proliferation, differentiation, Single Cell Raman Spectroscopy. 


\section{Abstract}

Dental pulp stem cells (DPSCs) are increasingly being recognized as a viable cell source for regenerative medicine. However, significant heterogeneity in their ex vivo expansion capabilities are well-established, which influences their regenerative and therapeutic potentials. As highly proliferative/multi-potent DPSCs are minority sub-populations within dental pulp, the development of non-invasive strategies capable of successfully discriminating between DPSC sub-populations with contrasting proliferative and differentiation capabilities in situ, would be immensely beneficial for the selective screening/isolation of superior quality DPSCs for in vitro assessment and therapy development. Consequently, this study assessed the effectiveness of Single Cell Raman Spectroscopy (SCRM), in distinguishing between DPSC sub-populations with contrasting proliferative and differentiation capabilities isolated from dental pulp tissues. Individual DPSC sub-populations were isolated from human third molars and identified as high or low proliferative and multi-potent or uni-potent, following in vitro expansion and senescence confirmation. High proliferative/multi-potent DPSCs, such as A3 (18PDs and 60PDs) and low proliferative/uni-potent DPSCs, including A1 and B1 (8PDs and 7PDs respectively), were analysed using an iHR550 Raman Spectrometer, equipped with a CCD camera and Eclipse TiU Inverted Microscope. Single cell spectra were acquired for 20 cells in each sub-population (10 spectra per nuclear and 10 spectra per cytoplasmic/membrane regions in each cell analysed), over 500-2100 $\mathrm{cm}^{-1}$. Spectra and peak assignments were obtained, followed by PCA and multivariate statistical analysis. Although DPSC spectra contained typical Raman peaks for nucleic acids, proteins and lipids, the spectral intensities of high proliferative/multi-potent DPSCs, A3 (18PDs), were higher than A3 (60PDs), A1 (8PDs) and B1 (7PDs), reflecting significantly elevated DNA $\left(729 \mathrm{~cm}^{-1}\right)$ and protein $\left(1111,1167,1245\right.$ and $\left.1680 \mathrm{~cm}^{-1}\right)$ contents overall. PCA and multivariate analysis revealed significant variations in scatter plots and Raman signatures, with distinct fingerprints for high proliferative/multi-potent DPSCs, A3 at 18PDs and 60PDs; versus the similar overlapping profiles for low proliferative/uni-potent DPSCs, A1 
(8PDs) and B1 (7PDs). This study confirms that SCRM successfully discriminates between DPSC sub-populations with contrasting proliferative and differentiation capabilities, advocating its further assessment as a viable technique for the selective non-invasive screening, identification and isolation of high proliferative/multi-potent DPSCs from dental pulp tissues for regenerative medicine applications.

\section{Impact Statement}

This study is the first to investigate and confirm the effectiveness of Single Cell Raman Spectroscopy (SCRM), in its ability to discriminate between DPSCs with contrasting proliferative and differentiation capabilities. The findings show that SCRM can rapidly and noninvasively distinguish and identify DPSC sub-populations in vitro with superior proliferative and multi-potency properties, versus lesser quality DPSCs; thereby overcoming the significant heterogeneity issues surrounding DPSC ex vivo expansion and differentiation capabilities. Such findings support further SCRM assessment for the selective screening/isolation of superior quality DPSCs from whole dental pulp tissues, for more effective in vitro evaluation and therapy development. 


\section{Introduction}

Tissue repair and regeneration in patients with chronic diseases are a major clinical challenge, especially with ever-increasing ageing populations worldwide. Adult mesenchymal stem cells (MSCs) are vital to tissue engineering for the development of cell-based therapies. Dental pulp stem cells (DPSCs) are increasingly recognized as a viable stem cell source for regenerative medicine purposes, due to their self-renewal, clonogenicity and multi-potent differentiation properties. $^{1-4}$ However, similar to bone marrow-derived MSCs, DPSCs possess significant heterogeneity, with individual clones demonstrating differences in proliferation and lineage differentiation capabilities. Indeed, despite heterogeneous DPSC population expansion achieving $>120$ population doublings (PDs) in vitro, only $20 \%$ of purified DPSCs are capable of proliferating > 20PDs. Of these, only two-thirds generated ectopic dentine in vivo, implying that subset DPSC populations differ in their regenerative potentials. ${ }^{5-6}$

DPSCs are also proposed to exist within distinct niches within dental pulp tissues (subodontoblast layer, pulpal vasculature and central pulp), ${ }^{7}$ which increases the complex nature of the origins and regenerative characteristics of individual DPSC sub-populations further. Such considerations are pivotal to the exploitation of DPSCs, as a significant limitation of MSC-based therapies is that extensive in vitro expansion eventually leads to proliferative decline and senescence, accompanied by impaired cellular regenerative properties. ${ }^{8-10}$ Despite significant differences in the ex vivo expansion capabilities of individual DPSCs being recognized, only recently has work begun to address the impact of such variations in proliferative capabilities prior to senescence, on the multi-potency of different DPSC sub-populations. ${ }^{11}$ Although high proliferative DPSCs achieved >80PDs, low proliferating DPSCs only completed <40PDs before senescence, correlating with DPSCs with high proliferative capacities possessing longer telomeres $(>18 \mathrm{~kb})$ than less proliferative populations $(5-13 \mathrm{~kb})$. Low proliferative DPSC senescence was also associated with early loss of stem cell marker characteristics and impaired osteogenic and chondrogenic differentiation, in favour of adipogenesis. In contrast, high 
proliferative DPSCs retained multi-potent differentiation capabilities, only demonstrating impaired differentiation following prolonged in vitro expansion (>60PDs). ${ }^{11}$

It has been proposed that high proliferative/multi-potent DPSCs are minority subpopulations within dental pulp tissues. ${ }^{7,} 11$ Therefore, in order to fully exploit these desirable DPSCs for regenerative purposes, it is imperative that strategies are developed which permit the optimisation of population selection through the selectively screening and isolation of superior quality DPSCs from dental pulp tissues for in vitro expansion, assessment and prudent cell banking; thereby aiding the translational development of more effective DPSC-based therapies for clinical evaluation and application. ${ }^{12-14}$ However, conventional ex vivo cellular expansion and stem cell/senescence characterisation techniques used to identify superior DPSCs are often expensive, labour-intensive and time-consuming; whilst cellular expansion can also detrimentally alter stem cell characteristics leading to reduced regenerative properties. ${ }^{11,15} \mathrm{Such}$ issues may be confounded by the requirement for invasive fixation, staining or cell permeabilisation techniques, which damage cellular components during MSC characteristation. ${ }^{15-18}$ Furthermore, these procedures prevent the reuse of evaluated cells, while many techniques utilised have resolution limitations and thus, cannot be performed at single cell levels. Consequently, there is a need to develop more non-destructive approaches to cell imaging, analysis and identification in situ, to provide highly sensitive real-time imaging and chemical information during cell characterisation; ultimately leading to the more effective use of MSCs for regenerative therapies.

Raman Spectroscopy is increasingly becoming a powerful analytical method in biomedical research, particularly due to its label-free and non-invasive characteristics. Raman Spectroscopy is a laser-based scattering technique, which permits the analysis of cellular chemical composition (DNA, RNA, lipids, proteins), molecular markers and the biological functions of individual cells within a spectral region of $600-2000 \mathrm{~cm}^{-1}$; through the use of Principal Component Analysis (PCA) and multivariate statistical analysis. ${ }^{19-20}$ Consequently, 
Raman spectra obtained for individual cells provide unique biochemical fingerprints of cellular status and functions, including metabolism, phenotype, apoptosis/senescence; and abilities to distinguish between different cell types. Raman Spectroscopy is now widely used in regenerative medicine for the characterisation of multi-potency/pluri-potency and the differentiation status of MSCs from numerous sources, including dental pulp. ${ }^{16-18,21-23}$ However, this proof-of-concept study is the first to apply Single Cell Raman Spectroscopy (SCRM) to DPSC sub-populations to evaluate its abilities in providing real-time evidence capable of discriminating between DPSCs with contrasting proliferative and differentiation capabilities ex vivo. Successful validation will subsequently form the basis for the further assessment of SCRM for the identification and isolation of high proliferative/multi-potent DPSCs from intact dental pulp tissues in situ, for regenerative medicine purposes.

\section{Materials and Methods}

Dental pulp stem cell isolation and characterisation of proliferation/differentiation capabilities DPSCs were isolated from third molar teeth collected from patients (all female, age 18-30 years), with informed patient consent and ethical approval, as previously described. ${ }^{11}$ Single cell suspensions of dental pulp tissues were obtained, with DPSCs preferentially selected and isolated from cell suspensions by differential fibronectin adhesion assay. ${ }^{11,24}$ Isolated cells were confirmed as DPSCs through cell surface marker expression (positive for MSC markers, CD73, CD90 and CD105; negative for hematopoietic stem cell marker, CD45). DPSCs subsequently underwent extended culture expansion and characterisation as being high or low proliferative and multi- or uni-potent. Proliferation analysis was based on the PDs reached by each DPSC sub-population prior to senescence (at <0.5PDs/week); and the detection of other senescencerelated markers, including reduced telomere lengths, positive senescence-associated $\beta$ galactosidase staining; and increased p53, p2 $1^{\text {waf1 }}$ and p16 ${ }^{\mathrm{INK} 4 \mathrm{a}}$ expression..$^{11}$ Differentiation analyses were based on the abilities of each DPSC sub-population to undergo osteogenic, 
chondrogenic and adipogenic differentiation, via detection of established differentiation markers as previously described. ${ }^{11}$ Individual DPSC sub-populations were subsequently confirmed as being high proliferative/multi-potent DPSCs (such as A3, capable of >80PDs) or low proliferative/uni-potent DPSCs (such as A1 and B1, capable of <40PDs). ${ }^{11}$

\section{Raman Spectroscopy instrumentation}

The Raman Microscopy system used in this study is shown in Fig. 1. The instrument was based on an Eclipse Ti-U Inverted Microscope, with a 20x $(\mathrm{NA}=0.75)$ dry objective (Nikon, Kingston upon Thames, UK). The laser source was a Diode Pumped Solid-State Laser, operating at 532 $\mathrm{nm}$ and maximum power of $50 \mathrm{~mW}$ (Lasever, Ningbo, China). The laser beam passed through a Semrock Clean-up Filter (Laser 2000, Huntington, UK), to remove optical noise. To fulfil the back-aperture (diameter, $4.37 \mathrm{~mm}$ ) of the 20x objective, the laser beam size $\left(1 / \mathrm{e}^{2}\right)$ of $2 \mathrm{~mm}$ was expanded x3. At this size, a 1:3 beam expander composed of two visible achromatic lenses of focal lengths, $f=+75 \mathrm{~mm}$ and $\mathrm{f}=-25 \mathrm{~mm}$, were used with a scan lens of $\mathrm{f}=100 \mathrm{~mm}$ (all Thorlabs, Ely, UK).

The laser beam was reflected from a Semrock Dichroic Beam Splitter (Laser 2000) and focused onto each sample by the objective lens. Raman scattered light was collected by the objective lens and transmitted via the same Dichroic Beam Splitter. Scattered light passed through a Steinheil Triplet Achromatic Lens (1:1 magnification, $f=50 \mathrm{~mm}$, Edmund Optics, York, UK); followed by a 6mm Confocal Slit (Thorlabs) and another Triplet Achromatic Lens. A bi-convex lens ( $\mathrm{f}=25 \mathrm{~mm}$, Thorlabs) was placed in front of the Confocal Slit to image samples onto the grating of the spectrometer. Before entering the Spectrometer, Raman scattered light passed through a Semrock Long Pass Filter (Laser 2000), to reject Rayleigh scatter.

The spectrometer used was an iHR550 Jobin Yvon Imaging Spectrophotometer (Horiba, Kyoto, Japan), equipped with a 600 grooves $/ \mathrm{mm}$ grating (spectral resolution, $4 \mathrm{~cm}^{-1}$ ) and Newton 940 Spectroscopy CCD Camera (Andor Technology, Belfast, UK). The spectrometer was 
calibrated prior to each experiment, using a clear Neon Lamp (RS Components, Corby, UK), which allowed Raman spectra pixel conversion to wavenumbers. Neon spectra were taken at central wavelength positions of $600 \mathrm{~nm}$. Neon spectra in pixels were compared with published neon spectra in wavelength format; and pixel-wavelength experimental data plotted using linear regression. Detector sensitivity was determined to be $\sim 98 \%$.

\section{Sample preparation and Single Cell Raman Spectroscopy analysis}

High proliferative/multi-potent DPSC sub-population, A3, at early (18PDs) and late (60PDs) stages in their proliferative lifespans; and low proliferative/uni-potent DPSCs, A1 and B1 (8PDs and 7PDs, respectively); were cultured in T-75 flasks until 80\% confluence and harvested using StemPro ${ }^{\circledR}$ Accutase ${ }^{\circledR}$ (ThermoFisher Scientific, Paisley, UK). Cells were retrieved, washed (x2) in phosphate buffered saline and fixed in $96 \%$ alcohol, at $2.5 \times 10^{5}$ cells $/ \mathrm{mL}$. Sample chambers were made using $80 \mu \mathrm{m}$ deep vinyl spacers between quartz slides and coverslips ( $1 \mathrm{~mm}$ and 200 $\mu \mathrm{m}$ thickness, respectively; UQG, Cambridge, UK). DPSC suspensions $\left(5 \times 10^{3}\right.$ cells, $\left.20 \mu \mathrm{L}\right)$ were pipetted directly onto the quartz surfaces and DPSCs allowed to sediment onto the inverted coverslips for $\sim 30 \mathrm{~min}$, prior to analysis. SCRM spectra were acquired from 20 fixed cells from each DPSC sub-population, along with the background spectra. The laser power on each sample during signal acquisition was $\sim 36 \mathrm{~mW}$; and each spectrum was collected in the range from 500 $2100 \mathrm{~cm}^{-1}$ (grating 150 grooves $/ \mathrm{mm}$ ) with an integration time of $60 \mathrm{~s}$. At the beginning of each Raman session, the dark current of the Andor CCD camera was also quantified. Ten spectra were measured for the nuclear and ten for the cytoplasmic/membrane regions of each cell analysed, to obtain average spectra representative of each DPSC sub-population analysed.

\section{Single Cell Raman Spectroscopy data analysis}

Raw data were converted into Raman spectra using Origin V7 Software (OriginLab, Northampton, USA). Mean Raman and background spectra derived from the 20 individual cells, 
were collected and dark currents subtracted. Average backgrounds were subtracted from each mean Raman spectrum for fluorescence suppression. ${ }^{25}$ All spectra were normalised to peak areas at $1450 \mathrm{~cm}^{-1}$ (related to $\mathrm{C}-\mathrm{H}$ deformation vibrations in nucleic acids, proteins, carbohydrates and lipids), to enable direct data comparisons ${ }^{26}$. Data were detected as relative intensities (arbitrary units, a.u.), with the main Raman spectra peaks for each DPSC sub-population assigned through comparisons to previously described reference peaks. ${ }^{16,27-28}$ Raman spectra were further analysed using PCA and multivariate statistical analysis, with principal components (PC) scores reflecting the most significant variations in DPSC Raman spectra. PC analysis was performed using built-in $\mathrm{R}$ function, prcomp .

\section{Statistical analysis}

The peak intensities of selected bands for the high proliferative/multi-potent and low proliferative/uni-potent DPSCs, were expressed as mean \pm standard deviation of the mean (SD). Statistical analysis was performed using Prism Software (GraphPad Software, San Diego, USA), with statistical significance between selected Raman peaks confirmed by unpaired Student's t tests. Significance was considered at $\mathrm{p}<0.05$.

\section{Results}

Discrimination between dental pulp stem cells with contrasting proliferative/differentiation capabilities

Distinct DPSC sub-populations were previously isolated and characterised as high proliferative/multi-potent (A3) and low proliferative/uni-potent (A1 and B1). ${ }^{11}$ DPSCs were subsequently analysed to confirm whether SCRM could distinguish between such subpopulations with contrasting proliferative and differentiation capabilities in vitro, based on the respective Raman spectra and molecular fingerprints obtained. 
Mean Raman spectra obtained over $600-1800 \mathrm{~cm}^{-1}$, for particular high proliferative/multi-potent and low proliferative/uni-potent DPSC sub-populations at specific PDs, are shown in Fig. 2. The dominant peaks and corresponding assignments identified within these Raman spectra, are presented in Table 1. Spectra for high proliferative/multi-potent DPSC sub-population, A3, at early (18PDs) and late (60PDs) stages in their proliferative lifespans; and low proliferative/uni-potent DPSCs, A1 and B1 (8PDs and 7PDs, respectively), were identified to contain typical Raman peaks for biomolecules, including DNA and proteins, in addition to the expected detection of $\mathrm{C}-\mathrm{H}$ deformation vibrations in nucleic acids, proteins, carbohydrates and lipids at $1450 \mathrm{~cm}^{-1}$; consistent with reports with various other cell types. ${ }^{15-16,27-28}$ Specifically, Raman bands associated with DNA bases at $729 \mathrm{~cm}^{-1}$ (ring breathing of adenine) and $765 \mathrm{~cm}^{-1}$ (pyrimidine ring breathing mode); and the nucleic acid backbone at 789 and $1092 \mathrm{~cm}^{-1}$ ( $\beta$ conformation DNA and $\mathrm{PO}_{2}^{-}$phosphodiester stretching vibrations) were identified. Similarly, Raman protein bands were detected at 834, 858, 1167, 1245, 1402, 1629 and $1680 \mathrm{~cm}^{-1}$, accounting for tyrosine, isoleucine, amide III, C $\beta$ rocking (arginine), serine and amide I regions, respectively. Regions 1-5 denote the largest peaks detected (Fig. 2 and Table 1, bracketed regions). However, spectral differences were particularly evident when comparing high proliferative/multi-potent DPSC sub-population, A3 at 18PDs, versus A3 at 60PDs and the low proliferative/uni-potent sub-populations, A1 (8PDs) and B1 (7PDs). Indeed, the spectral intensities of A3 (18PDs) were much higher than A3 (60PDs), A1 (8PDs) and B1 (7PDs), reflecting elevated DNA and protein contents overall. Furthermore, peaks for RNA ribose C-O vibration $\left(1054 \mathrm{~cm}^{-1}\right)$ were only identifiable for high proliferative/multi-potent sub-population, A3 (18PDs, Fig. 2 and shaded region, Table 1). Other notable variations identified included the increased detection of benzoid ring deformation $\left(1111 \mathrm{~cm}^{-1}\right)$ with high proliferative/multi-potent sub-population, A3 (18PDs) only; and phenylalanine peaks $\left(958 \mathrm{~cm}^{-1}\right)$ with high proliferative/multi-potent sub-population, A3, at both 18PDs and 60PDs only. Sub-population differences in other amino acid and protein levels were also detected, with the absence of peaks 
relating to $\mathrm{C}-\mathrm{C} / \mathrm{C}-\mathrm{N}$ protein stretching $\left(1155 \mathrm{~cm}^{-1}\right)$ and tyrosine $\left(1614 \mathrm{~cm}^{-1}\right)$, with low proliferative/uni-potent sub-population, B1 (7PDs, Fig. 2 and shaded regions, Table 1).

\section{Raman peak intensity differences related to cell proliferation and growth}

To further illustrate spectral differences between DPSC sub-populations, the peak intensity differences in selected DNA and protein bands associated with cellular proliferation/growth, were assessed between high proliferative/multi-potent sub-population, A3 at 18PDs and 60PDs, compared to low proliferative/uni-potent sub-populations, A1 (8PDs) and B1 (7PDs, in bold, Table 2). In line with their more proliferative nature, DPSC sub-population, A3 (18PDs), exhibited higher DNA (729, 786 and $\left.1092 \mathrm{~cm}^{-1}\right)$ and protein $\left(1167,1245,1629\right.$ and $\left.1680 \mathrm{~cm}^{-1}\right)$ peak intensities, particularly versus both low proliferative/uni-potent sub-populations, A1 (8PDs) and B1 (7PDs, Table 2). Such peak intensity differences were most prominent for reductions in DNA $\left(729,789\right.$ and $\left.1092 \mathrm{~cm}^{-1}\right)$ and $\mathrm{C}-\mathrm{H}$ deformation vibrations $\left(1450 \mathrm{~cm}^{-1}\right)$ in the low proliferative/uni-potent sub-populations, with peak intensity reductions even being of greater magnitude than those for high proliferative/multi-potent sub-population, A3 (60PDs). In contrast, high proliferative/multi-potent sub-population, A3 (60PDs), exhibited more subtle differences in peak intensities, versus their early PD counterparts (Table 2). However, A3 (60PDs) and low proliferative/uni-potent sub-populations, A1 (8PDs) and B1 (7PDs), all exhibited major decreases in protein-related peak intensities at $1167 \mathrm{~cm}^{-1}$ (isoleucine), $1245 \mathrm{~cm}^{-}$

${ }^{1}$ (amide III region), $1402 \mathrm{~cm}^{-1}$ (C $\beta$ rocking, arginine), $1629 \mathrm{~cm}^{-1}$ (serine); and $1680 \mathrm{~cm}^{-1}$ (amide I region), compared to high proliferative/multi-potent, A3 (18PDs, Table 2).

Peak intensity differences between high proliferative A3 (18PDs) and low proliferative A1 (8PDs) and B1 (7PDs) were confirmed to be statistically significant for DNA ( $729 \mathrm{~cm}^{-1}$, both $p<0.001)$ and protein $\left(1167,1245\right.$ and $\left.1680 \mathrm{~cm}^{-1}, p<0.001-p<0.05\right)$ peaks (Fig. 3). However, no significant differences were shown between A3 (18PDs) and both low proliferative/uni-potent 
sub-populations, A1 (8PDs) and B1 (7PDs), for DNA peaks at 786 and $1092 \mathrm{~cm}^{-1}$; and protein peaks at $1629 \mathrm{~cm}^{-1}($ all $p>0.05)$.

PCA discrimination between dental pulp stem cells with contrasting proliferative and differentiation capabilities

Further analysis of high proliferative/multi-potent and low proliferative/uni-potent DPSC Raman spectra using PCA and multivariate statistical analysis, revealed spectral discrimination between DPSC sub-populations. This was particularly evident with the loading plots for principle components, PC3 and PC4, which provided the largest variance $(\mathrm{PC} 3=98 \%, \mathrm{PC} 4=97 \%)$, between DPSC sub-population spectra (Fig. 4). 2D scatter plot obtained using PC3 and PC4 scores for high proliferative/multi-potent DPSC sub-population, A3, at 18PDs and 60PDs; and low proliferative/uni-potent DPSCs, A1 and B1 (8PDs and 7PDs, respectively); are shown in Fig. 5. The scatter plots demonstrated clear spatial separation between the profiles obtained for each DPSC sub-population, with three clearly distinct clusters evident. Furthermore, the compact nature of individual clusters indicated high levels of homogeneity within each dataset. These clusters were particularly representative of high proliferative/multi-potent DPSC, A3 (18PDs, in blue) and (60PDs, in orange); and the low proliferative/uni-potent DPSCs, A1 and B1 (8PDs and 7PDs, in red and green, respectively). The clusters for both low proliferative/unipotent DPSCs, A1 (8PDs) and B1 (7PDs) showed almost complete overlap indicative of high Raman signature similarity. In contrast, profiles for the high proliferative/multi-potent DPSC, A3 (at 18PDs and 60PDs), were distinctly dissimilar to those for A1 (8PDs) and B1 (7PDs), as these exhibited well separated signatures with no A1 or B1 cluster overlap. Furthermore, despite being derived from the same DPSC sub-population, both A3 profiles did not exhibit any overlap when compared at early (18PDs) and late (60PDs) stages in their proliferative lifespans, indicative of large variations in DPSC sub-population signatures overall. 


\section{Discussion}

The present study describes a novel investigation, confirming the abilities of SCRM to noninvasively discriminate between DPSC sub-populations with contrasting proliferative and differentiation capabilities. The results obtained also provide unique insight into the biochemical differences which exist between non-senescent and senescent DPSC sub-populations.

Previous reports have demonstrated that only $20 \%$ of purified DPSCs undergo >20PDs, compared with heterogeneous DPSC populations (>120PDs), highlighting the presence of DPSC sub-populations in dental pulp tissues with contrasting proliferative and differentiation capabilities. ${ }^{5-7}$ Consistent with such findings, recent studies have reported key differences between DPSC sub-populations in the relative susceptibilities to replicative (telomeredependent) senescence, correlating with contrasting differentiation capabilities within each population. ${ }^{11}$ Whilst highly proliferative DPSCs, A3, reached >80PDs before undergoing senescence, low proliferative DPSCs, A1 and B1, only achieved <40PDs. Unlike low proliferative DPSCs, highly proliferative DPSCs retained stem cell marker expression and multipotent differentiation capabilities, which were only impaired at much later PDs (>60PDs). Therefore, it has been proposed that such high proliferative DPSCs are responsible for the extensive expansion potential of heterogeneous populations (>120PDs) in vitro, ${ }^{5-6}$ as less proliferative, uni-potent DPSCs would be selectively lost from the mixed populations during extended sub-culture. ${ }^{11,24}$

From the findings reported, it is evident that such phenotypic differences in DPSC subpopulation proliferative and regenerative capabilities can be rapidly and sensitively distinguished using SCRM, given the contrasting Raman fingerprints obtained for high proliferative/multipotent and low proliferative/uni-potent DPSCs as a consequence of distinct differences in the biochemical (DNA, RNA, protein, lipid) compositions of these cells overall. Such conclusions also reiterate the significant variations in the biological status and reparative properties of these DPSC sub-populations. ${ }^{11}$ Although Raman Spectroscopy has been used extensively in MSC 
research, studies have primarily focussed on its application to distinguish MSC populations from non-somatic cells; and the assessment of differentiation status in MSCs from various sources. ${ }^{16-}$ 18, 21-23 However, concurrent with its successful utilisation in the present study to discriminate between high proliferative/multi-potent and low proliferative/uni-potent DPSCs, Raman Spectroscopy has recently been shown to distinguish between proliferative/non-proliferative, senescent/non-senescent, young/aged and viable/non-viable cells, within other MSC and fibroblast populations. ${ }^{15,29-33}$

The increased spectral intensities obtained for high proliferative/multi-potent DPSC subpopulation, A3 (18PDs), compared to more senescent A3 (60PDs) and to low proliferative/unipotent DPSCs, A1 and B1 (8PDs and 7PDs, respectively), are consistent with recent studies reporting alterations in the biomolecular compositions of senescent fibroblast populations, versus non-senescent counterparts; relating to band intensities and/or band positions. ${ }^{31-32}$ Specifically, such studies have associated reductions in DNA $\left(788,1580 \mathrm{~cm}^{-1}\right)$ and protein $(1658$ $\left.\mathrm{cm}^{-1}\right)$ peaks, in addition to elevated lipid $\left(1732,2850\right.$ and $\left.2930 \mathrm{~cm}^{-1}\right)$ peak contents during cellular senescence, with protein and lipid modifications predominating overall. ${ }^{31-32}$ Amide I peak intensities have been demonstrated to be particularly sensitive to conformational changes during senescence, whilst distinct amide III region changes having also been reported..$^{31-32}$ Changes in the ratio of $1157 \mathrm{~cm}^{-1}(\mathrm{C}-\mathrm{C} / \mathrm{C}-\mathrm{N}$ protein stretching $)$ and $1174 \mathrm{~cm}^{-1}(\mathrm{C}-\mathrm{H}$ bending vibrations in phenylalanine and tyrosine) due to reduced $1157 \mathrm{~cm}^{-1}$ peak intensities, have further been proposed as characteristic signatures of umbilical cord-derived, MSC senescence. ${ }^{15}$ However, despite senescence-induced shifts in lipid conformation $\left(2850 \mathrm{~cm}^{-1}\right)$ having been assigned to the $\mathrm{CH}_{2}$ symmetric stretch of lipids, ${ }^{31}$ no such changes were evident herein, as SCRM spectra were recorded over $600-1800 \mathrm{~cm}^{-1}$.

From the assignments and the peak intensities identified, it was apparent that such differences reflected elevations in DNA $\left(729,786,1092\right.$ and $\left.1450 \mathrm{~cm}^{-1}\right)$ and protein $(1167,1245$, 1402, 1450, 1629 and $1680 \mathrm{~cm}^{-1}$ ) contents in non-senescent, high proliferative/multi-potent 
DPSC, A3 (18PDs). Such peak intensity increases were particularly significant for A3 (18PDs), at $729 \mathrm{~cm}^{-1}$ (ring breathing of adenine), $1111 \mathrm{~cm}^{-1}$ (benzoid ring deformation), $1167 \mathrm{~cm}^{-1}$ (isoleucine), $1245 \mathrm{~cm}^{-1}$ (amide III region), and $1680 \mathrm{~cm}^{-1}$ (amide I region). Additionally, subpopulation differences in other amino acid and protein contents were also detected, including increased $958 \mathrm{~cm}^{-1}$ (phenylalanine) peaks in high proliferative/multi-potent sub-population, A3, at $18-60 \mathrm{PDs}$ and 60PDs, respectively; in addition to reduced $1155 \mathrm{~cm}^{-1}(\mathrm{C}-\mathrm{C} / \mathrm{C}-\mathrm{N}$ protein stretching) and $1614 \mathrm{~cm}^{-1}$ (tyrosine) peaks with low proliferative/uni-potent sub-population, B1 (7PDs) only. As DNA, amino acids and proteins are fundamental biochemical constituents which facilitate cellular responses, contrasting intensities in these peaks are consistent with the proliferative and differentiation capabilities of high and low DPSC sub-populations overall. ${ }^{11,15 \text {, }}$ 31-34 Therefore, as Raman signature changes during senescence are proposed to be principally caused by biomolecular modifications to proteins and lipids, rather than changes in molecular abundance, ${ }^{31}$ the present findings are partly counterintuitive to such findings, considering the considerable reductions in biomolecular abundance evident herein. ${ }^{15}$ However, the particular predominance of amino acid/protein peak intensity changes between high/multi-potent and low proliferative/uni-potent DPSCs, concurs with previous reports. ${ }^{15,}$ 31-32

Senescence-induced biomolecular modifications are strongly linked with oxidative stress, due to elevated reactive oxygen species (ROS) production by senescent cells causing accumulative damage to cellular components. ${ }^{8,35-36}$ Although DNA is a strong candidate for oxidative modification, proteins and lipids are also highly susceptible to oxidative stressinduced, damage. ${ }^{37-39}$ Indeed, protein oxidation by ROS is mediated via amino acid modification, leading to increased cross-linking, aggregation and cleavage; which subsequently influence protein structure and function. ${ }^{40-41}$ ROS modification of amino acids results in the formation of numerous oxidised by-products, although many aromatic (phenylalanine, tyrosine) and non-aromatic (proline, histidine, lysine, arginine, serine) amino acids are known to be highly susceptible to ROS modification. ${ }^{40-41}$ Thus, it is conceivable that reductions in phenylalanine 
(958 $\left.\mathrm{cm}^{-1}\right)$, benzoid ring deformation $\left(1111 \mathrm{~cm}^{-1}\right)$, arginine $\left(1402 \mathrm{~cm}^{-1}\right)$, tyrosine $\left(1614 \mathrm{~cm}^{-1}\right)$ and serine $\left(1629 \mathrm{~cm}^{-1}\right)$ peaks in low proliferative/uni-potent DPSCs (A1, 8PDs; B1, 7PDs), are consequences of increased oxidative modification to these amino acids in low proliferative (senescent) sub-populations; leading to subsequent reductions in protein band detection (1155, 1245 and $\left.1680 \mathrm{~cm}^{-1}\right)$. Consequently, the spectral differences identified overall may be attributed to alterations in the quantities of certain biomolecular species between high/multi-potent and low proliferative/uni-potent DPSC sub-populations, in addition to conformational changes in protein, DNA, and lipid structures due to oxidative stress. ${ }^{15,31-32}$

PCA analysis was further capable of discriminating between high proliferative/multipotent DPSCs (A3, 18PDs) and the similarly overlapping clusters of low proliferative/uni-potent DPSCs, A1 (8PDs) and B1 (7PDs), coinciding with the proliferative and differentiation heterogeneity of DPSC sub-populations previously described. ${ }^{11}$ In particular, PC3 and PC4 components showed significant cluster separation between highly proliferative DPSC, A3, at early (18PDs) and late (60PDs) stages in their proliferative life-spans. As previous studies have also identified significant spatial separation between the scatter plots of non-senescent and senescent cell populations, ${ }^{31-32}$ obvious differences between the clusters for high (A3, 18PDs) and low proliferative/uni-potent DPSCs, A1 (8PDs) and B1 (7PDs) were shown, despite A1 and B1 being derived from different patient donors. As with the apparent DNA and protein peak intensity differences identified between the same DPSC sub-population, A3, at 18PDs and 60PDs; similar non-overlapping cluster differences were also observed. However, the A3 (60PDs) cluster remained well-separated from the clusters for low proliferative/uni-potent DPSCs, A1 (8PDs) and B1 (7PDs), with no overlap evident. Such variations in the A3 (60PDs) signatures to those of $\mathrm{A} 3$ (18PDs) and A1 (8PDs)/B1 (7PDs), may be interpreted as the detection of increased A3 transition from proliferation (18PDs) towards senescence (60PDs), although the A3 (60PDs) signatures had yet reached the equivalent senescence status displayed by A1 (8PDs) and B1 (7PDs). Indeed, despite good cluster separation between young and senescent cells, 
previous studies have further shown cluster overlap during senescence transition at intermediate PDs, indicating non-linear signature/peak intensity changes and individual cell heterogeneity within a population during senescence transition. ${ }^{15,31-32}$ However, we can only speculate on the underlying reasons for such differences in the cluster profiles and proliferative/differentiation responses between DPSC sub-populations at present, as intrinsic factors, such as those associated with patient donor characteristics, and/or the developmental origins and stem cell niche sources of these DPSC sub-populations within pulpal tissues, warrant additional consideration. ${ }^{5-7,11}$

Nonetheless, this study shows that SCRM can successfully and reliably discriminate between high proliferative/multi-potent and low proliferative/uni-potent DPSCs, with clear differences in biochemical signatures between these distinct DPSC sub-populations. Therefore,

concomitant with their established heterogeneous proliferative and regenerative properties, ${ }^{5-6,11}$ such contrasting SCRM profiles reflect the high proliferative/multi-potent nature of certain DPSCs, evident by their increased DNA/protein contents and the prevalence of particular DNA and protein signature peaks, compared to low proliferative/uni-potent DPSCs. As these signature differences may reflect similarly contrasting proliferative and differentiation characteristics between DPSC sub-populations in vivo, this study advocates further SCRM assessment as a viable tool for the selective screening and isolation of desirable high proliferative/multi-potent DPSCs from dental pulp tissues for regenerative medicine applications.

\section{Acknowledgements}

This work was supported by PhD studentship funding, awarded to Dr Amr Alraies by Albawani Company, Saudi Arabia.

\section{Disclosure Statement}

No competing financial interests exist. 


\section{References}

1. Ledesma-Martínez, E., Mendoza-Núñez, V.M., and Santiago-Osorio, E. Mesenchymal stem cells derived from dental pulp: A review. Stem Cells Int 2016, 4709572, 2016.

2. Nuti, N., Corallo, C., Chan, B.M., Ferrari, M., and Gerami-Naini, B. Multipotent differentiation of human dental pulp stem cells: A literature review. Stem Cell Rev 12, 511, 2016

3. Chalisserry, E.P., Nam, S.Y., Park, S.H., and Anil, S. Therapeutic potential of dental stem cells. J Tissue Eng 8, $2041731417702531,2017$.

4. Anitua, E., Troya, M., and Zalduendo, M. Progress in the use of dental pulp stem cells in regenerative medicine. Cytotherapy 20, 479, 2018.

5. Gronthos, S., Mankani, M., Brahim, J., Robey, P.G., and Shi, S. Postnatal human dental pulp stem cells (DPSCs) in vitro and in vivo. Proc Natl Acad Sci USA 97, 13625, 2000.

6. Gronthos, S., Brahim, J., Li, W., Fisher, L.W., Cherman, N., Boyde, A., DenBesten, P., Robey, P.G., and Shi, S. Stem cell properties of human dental pulp stem cells. J Dent Res 81, $531,2002$.

7. Sloan, A.J., and Waddington, R.J. Dental pulp stem cells: What, where, how? Int J Paediatr Dent 19, 61, 2009.

8. Campisi, J., and d'Adda di Fagagna, F. Cellular senescence: When bad things happen to good cells. Nat Rev Mol Cell Biol 8, 729, 2007.

9. Wagner, W., Ho, A.D., and Zenke, M. Different facets of aging in human mesenchymal stem cells. Tissue Eng Part B Rev 16, 445, 2010.

10. Li, Y., Wu, Q., Wang, Y., Li, L., Bu, H., and Bao, J. Senescence of mesenchymal stem cells (Review). Int J Mol Med 39, 775, 2017.

11. Alraies, A., Alaidaroos, N.Y., Waddington, R.J., Moseley, R., and Sloan, A.J. Variation in human dental pulp stem cell ageing profiles reflect contrasting proliferative and regenerative capabilities. BMC Cell Biol 18, 12, 2017. 
12. Hilkens, P., Driesen, R.B., Wolfs, E., Gervois, P., Vangansewinkel, T., Ratajczak, J., Dillen, Y., Bronckaers, A., and Lambrichts, I. Cryopreservation and banking of dental stem cells. Adv Exp Med Biol 951, 199, 2016.

13. Ferrúa, C.P., Centeno, E.G.Z., Rosa, L.C.D., Amaral, C.C.D., Severo, R.F., Sarkis-Onofre, R., Nascimento, G.G., Cordenonzi, G., Bast, R.K., Demarco, F.F., and Nedel, F. How has dental pulp stem cells isolation been conducted? A scoping review. Braz Oral Res 31, e87, 2017.

14. Rodas-Junco, B.A., and Villicaña, C. Dental pulp stem cells: Current advances in isolation, expansion and preservation. Tissue Eng Regen Med 14, 333, 2017.

15. Bai, H., Li, H., Han, Z., Zhang, C., Zhao, J., Miao, C., Yan, S., Mao, A., Zhao, H., and Han, Z. Label-free assessment of replicative senescence in mesenchymal stem cells by Raman microspectroscopy. Biomed Opt Expr 6, 4493, 2015.

16. Ghita, A., Pascut, F.C., Mather, M., Sottile, V., and Notingher, I. Cytoplasmic RNA in undifferentiated neural stem cells: A potential label-free Raman spectral marker for assessing the undifferentiated status. Anal Chem 84, 3155, 2012.

17. Hung, P.S., Kuo, Y.C., Chen, H.G., Chiang, H.H., and Lee, O.K. Detection of osteogenic differentiation by differential mineralized matrix production in mesenchymal stromal cells by Raman spectroscopy. PLoS One 8, e65438, 2013.

18. Suhito, I.R., Han, Y., Min, J., Son, H., and Kim, T.H. In situ label-free monitoring of human adipose-derived mesenchymal stem cell differentiation into multiple lineages. Biomaterials 154, 223, 2018.

19. Ichimura, T., Chiu, L.D., Fujita, K., Kawata, S., Watanabe, T.M., Yanagida, T., and Fujita, H. Visualizing cell state transition using Raman spectroscopy. PLoS One 9, e84478, 2014. 20. Ghita, A, Pascut, F.C., Sottile, V., Denning, C., and Notingher, I. Applications of Raman micro-spectroscopy to stem cell technology: Label-free molecular discrimination and monitoring cell differentiation. EPJ Tech Instrum 2, 6, 2015. 
21. Lee, Y.J., Vega, S.L., Patel, P.J., Aamer, K.A., Moghe, P.V., and Cicerone, M.T. Quantitative, label-free characterization of stem cell differentiation at the single-cell level by broadband coherent anti-Stokes Raman scattering microscopy. Tissue Eng Part C Methods 20, $562,2014$.

22. Mitchell, A., Ashton, L., Yang, X.B., Goodacre, R., Tomlinson, M.J., Smith, A., and Kirkham, J. Aseptic Raman spectroscopy can detect changes associated with the culture of human dental pulp stromal cells in osteoinductive culture. Analyst 140, 7347, 2015.

23. Salehi, H., Collart-Dutilleul, P.Y., Gergely, C., and Cuisinier, F.J. Confocal Raman microscopy to monitor extracellular matrix during dental pulp stem cells differentiation. J Biomed Opt 20, 076013, 2015.

24. Waddington, R.J., Youde, S.J., Lee, C.P., and Sloan, A.J. Isolation of distinct progenitor stem cell populations from dental pulp. Cells Tiss Organs 189, 268, 2009.

25. Mazilu, M., De Luca, A.C., Riches, A.C., Herrington, C.S., and Dholakia, K. Optimal algorithm for fluorescence suppression of modulated Raman spectroscopy. Opt Express 18, 11382, 2010.

26. Chan, J.W., Lieu, D.K., Huser, T., and Li, R.A. Label-free separation of human embryonic stem cells and their cardiac derivatives using Raman spectroscopy. Anal Chem 81, 1324, 2009. 27. Movasaghi, Z., Rehman, S., and Rehman, I.U. Raman spectroscopy of biological tissues. Appl Spectrosc Rev 42, 493, 2007.

28. Notingher, I. Raman Spectroscopy cell-based biosensors. Sensors 7, 1343, 2007.

29. Bai, H., Chen, P., Fang, L., Lin, G., Tang, Q., Mu, G.G., Gong, W., Liu, Z.P., Wu, H., Zhao, H., and Han, Z.C. Detecting viability transitions of umbilical cord mesenchymal stem cells by Raman micro-spectroscopy. Laser Phys Lett 8, 78, 2011.

30. Harkness, L., Novikov, S.M., Beermann, J., Bozhevolnyi, S.I., and Kassem, M. Identification of abnormal stem cells using Raman spectroscopy. Stem Cells Dev 21, 2152, 2012. 
31. Eberhardt, K., Beleites, C., Marthandan, S., Matthäus, C., Diekmann, S., and Popp, J. Raman and infrared spectroscopy distinguishing replicative senescent from proliferating primary human fibroblast cells by detecting spectral differences mainly due to biomolecular alterations. Anal Chem 89, 2937, 2017.

32. Eberhardt, K., Matthäus, C., Winter, D., Wiegand, C., Hipler, U.C., Diekmann, S., and Popp, J. Raman and infrared spectroscopy differentiate senescent from proliferating cells in a human dermal fibroblast 3D skin model. Analyst 142, 4405, 2017.

33. Eberhardt, K., Matthäus, C., Marthandan, S., Diekmann, S., and Popp, J. Raman and infrared spectroscopy reveal that proliferating and quiescent human fibroblast cells age by biochemically similar but not identical processes. PLoS One 13, e0207380, 2018.

34. Higuera, G.A., Schop, D., Spitters, T.W., van Dijkhuizen-Radersma, R., Bracke, M., de Bruijn, J.D., Martens, D., Karperien, M., van Boxtel, A., and van Blitterswijk, C.A. Patterns of amino acid metabolism by proliferating human mesenchymal stem cells. Tissue Eng Part A 18, $654,2012$.

35. Chaudhari, P., Ye, Z., and Jang, Y.Y. Roles of reactive oxygen species in the fate of stem cells. Antioxid Redox Signal 20, 1881, 2014.

36. Jeong, S.G., and Cho, G.W. Endogenous ROS levels are increased in replicative senescence in human bone marrow mesenchymal stromal cells. Biochem Biophys Res Commun 460, 971, 2015.

37. Evans, M.D., Dizdaroglu, M., and Cooke, M.S. Oxidative DNA damage and disease: Induction, repair and significance. Mutat Res 567, 1, 2004.

38. Levine, R.L., and Stadtman, E.R. Oxidative modification of proteins during aging. Exp Gerontol 36, 1495, 2001.

39. Spiteller, G. Lipid peroxidation in aging and age-dependent diseases. Exp Gerontol 36, $1425,2001$. 
40. Dean, R.T., Fu, S., Stocker, R., and Davies, M.J. Biochemistry and pathology of radicalmediated protein oxidation. Biochem J 324, 1, 1997.

41. Davies, M.J. Protein oxidation and peroxidation. Biochem J 473, 805, 2016.

Address correspondence to:

Ryan Moseley, PhD

Regenerative Biology Group

School of Dentistry

Cardiff Institute of Tissue Engineering and Repair (CITER)

College of Biomedical and Life Sciences

Cardiff University

$U K$

Email:MoseleyR@cardiff.ac.uk 


\section{Figure Legends}

FIG. 1. Schematic diagram of the Raman Microscopy set-up used in this study.

FIG. 2. Comparison of mean Raman spectral fingerprints for high proliferative/multi-potent DPSC sub-population, A3 at 18PDs and 60PDs; and low proliferative/uni-potent DPSC subpopulations, A1 and B1 (8PDs and 7PDs, respectively). Mean spectra were determined from $\mathrm{n}=20$ individual cells per DPSC sub-population and displayed in the fingerprint range of 600 $1800 \mathrm{~cm}^{-1}$. Spectra were normalised to peak areas at $1450 \mathrm{~cm}^{-1}$. Data were detected as relative intensities (arbitrary units, a.u.). The most prominent spectral bands are highlighted (in blue) and the corresponding wavenumbers assigned. Regions 1-5 denote the largest peaks detected. All spectra contained typical Raman peaks for DNA $\left(729,765,789\right.$ and $\left.1092 \mathrm{~cm}^{-1}\right)$, proteins $\left(834,858,1167,1245,1402,1629\right.$ and $\left.1680 \mathrm{~cm}^{-1}\right)$ and biomolecular C-H deformation vibrations $\left(1450 \mathrm{~cm}^{-1}\right)$. The spectral intensity of A3 at 18PDs were much higher than A3 (60PDs), A1 (8PDs) and B1 (7PDs), reflecting elevated DNA and protein contents overall. Certain peaks were particularly identifiable in high proliferative/multi-potent sub-population, A3, at 18PDs (see Table 1 for further details).

FIG. 3. Mean peak intensities for selected DNA $\left(729,789\right.$ and $\left.1092 \mathrm{~cm}^{-1}\right)$ and protein $(1167$, 1245, 1629 and $1680 \mathrm{~cm}^{-1}$ ) bands commonly associated with cellular proliferation/growth, detected in Raman spectra of high proliferative/multi-potent DPSC sub-population, A3 (18PDs); and low proliferative/uni-potent DPSC sub-populations, A1 and B1 (8PDs and 7PDs, respectively). Peak intensity data were presented as arbitrary units (a.u.). Statistically significant increases in peak intensities were shown for DNA $\left(729 \mathrm{~cm}^{-1}, p<0.001\right)$ and protein $(1167,1245$ and $\left.1680 \mathrm{~cm}^{-1}, p<0.001-p<0.05\right)$ peaks, in high proliferative/multi-potent DPSC sub-population, A3 (18PDs). No other significant differences were shown $(p>0.05)$. Peak intensity values were determined from $n=20$ individual cells per DPSC sub-population, with data expressed as mean \pm SD $(* p<0.05, * * p<0.01$ and $* * * p<0.001)$. 
FIG. 4. Principle Component Analysis (PCA) loading plots for (A) PC3 and (B) PC4, which provided the largest variance and spectral discrimination between high proliferative/multi-potent DPSC sub-population, A3 at 18PDs and 60PDs; and low proliferative/uni-potent DPSC subpopulations, A1 and B1 (8PDs and 7PDs, respectively). Extent of variation data were presented as arbitrary units (a.u.).

FIG. 5. Principle Component Analysis (PCA) 2D scatter plots obtained using PC3 and PC4 scores for individual Raman spectra for high proliferative/multi-potent DPSC sub-population, $\mathrm{A} 3$, at 18PDs and 60PDs; and low proliferative/uni-potent DPSC sub-populations, A1 and B1 (8PDs and 7PDs, respectively). Scatter plot demonstrated widespread differences between DPSC sub-population profiles, with three distinct Raman clusters evident for high proliferative/multi-potent DPSC, A3 at 18PDs (in blue), A3 at 60PDs (in orange); and low proliferative/uni-potent DPSCs, A1 and B1 at 8PDs and 7PDs (in red and green, respectively). Low proliferative/uni-potent DPSC clusters exhibited almost complete overlap, indicative of high signature similarity. Profiles for the high proliferative/multi-potent DPSC, A3 (18PDs) and (60PDs), were distinctly dissimilar to each other and to those for A1 (8PDs) and B1 (7PDs), indicative of large Raman signature differences overall. Spectra were determined from $n=20$ individual cells per DPSC sub-population. 
Table 1. Dominant peaks and their corresponding assignments, ${ }^{16,27-28}$ identified in mean Raman spectra for high proliferative/multi-potent DPSC sub-population, A3 at 18PDs and 60PDs; and low proliferative/uni-potent DPSC sub-populations, A1 and B1, (8PDs and 7PDs, respectively). Regions 1-5 (in bold) denote the largest detectable peaks. Shaded areas indicate peaks demonstrating the largest differences between DPSC sub-populations.

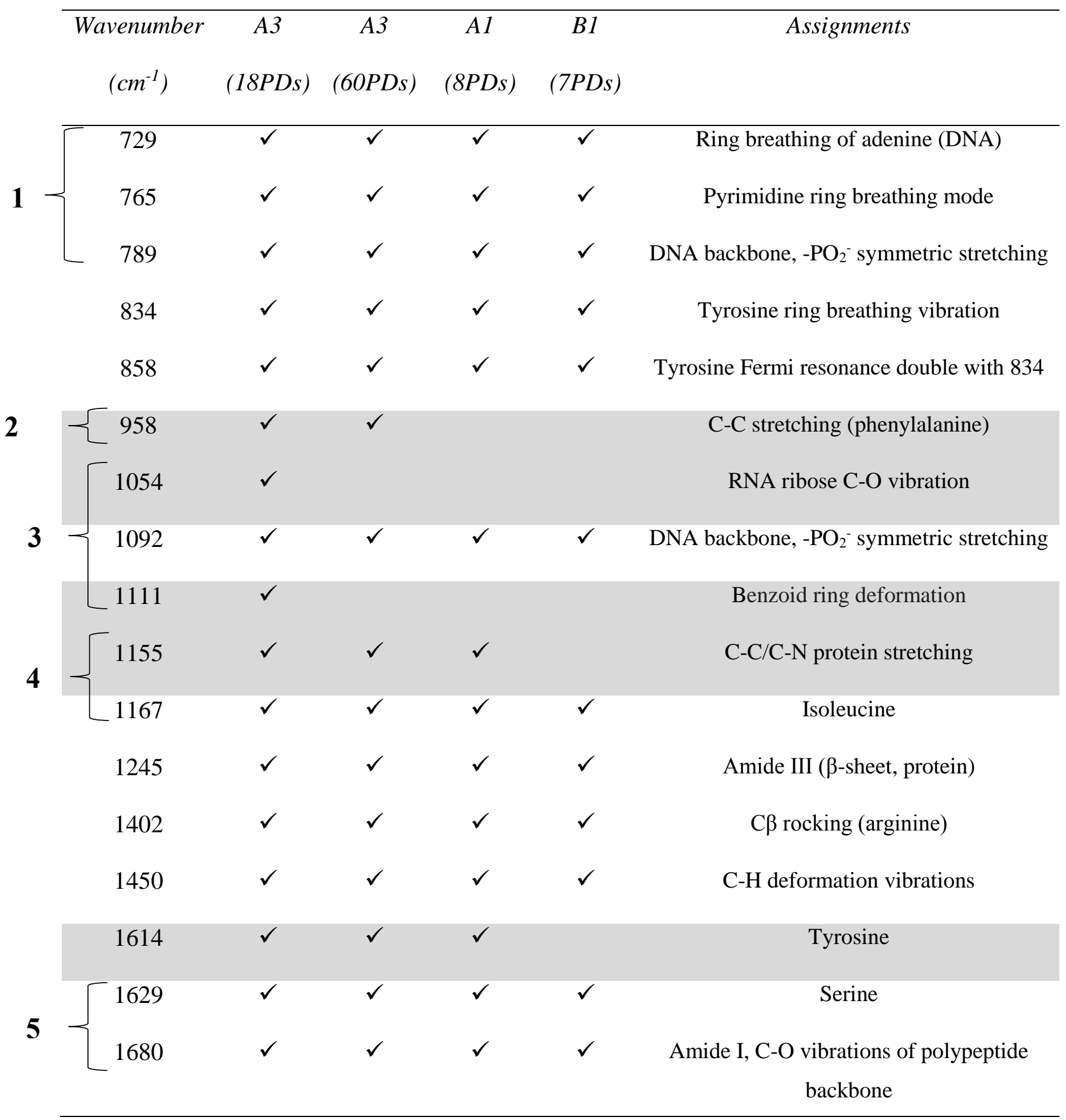


Table 2. Percentage intensity decreases in selected DNA and protein bands (in bold), detected in Raman spectra of high proliferative/multi-potent DPSC sub-population, A3 (60PDs) and low proliferative/uni-potent DPSC sub-populations, A1 and B1, (8PDs and 7PDs, respectively); compared to high proliferative/multi-potent DPSC sub-population, A3 (18PDs).

\begin{tabular}{|c|c|c|}
\hline Wavenumber $\left(\mathrm{cm}^{-1}\right)$ & Assignments & \% Decreases Versus A3 (18PDs) \\
\hline \multirow[t]{2}{*}{729} & Ring breathing of adenine & $\mathrm{A} 3(60 \mathrm{PDs})=\mathbf{6 . 0 \%} ; \mathrm{A} 1(8 \mathrm{PDs})=$ \\
\hline & (DNA) & $\mathbf{4 0 . 3 \%} ; \mathrm{B} 1(7 \mathrm{PDs})=\mathbf{3 9 . 9 \%}$ \\
\hline \multirow[t]{2}{*}{789} & DNA backbone, $-\mathrm{PO}_{2}^{-}$ & $\mathrm{A} 3(60 \mathrm{PDs})=\mathbf{1 0 . 2 \%} ; \mathrm{A} 1(8 \mathrm{PDs})=$ \\
\hline & symmetric stretching & $\mathbf{4 6 . 4 \%} ; \mathrm{B} 1(7 \mathrm{PDs})=\mathbf{3 1 . 3 \%}$ \\
\hline \multirow[t]{2}{*}{1092} & DNA backbone, $-\mathrm{PO}_{2}^{-}$ & $\mathrm{A} 3(60 \mathrm{PDs})=\mathbf{4 . 8 \%} ; \mathrm{A} 1(8 \mathrm{PDs})=$ \\
\hline & symmetric stretching & $\mathbf{3 0 . 2 \%} ; \mathrm{B} 1(7 \mathrm{PDs})=\mathbf{2 1 . 4 \%}$ \\
\hline \multirow[t]{2}{*}{1167} & Isoleucine & $\mathrm{A} 3(60 \mathrm{PDs})=\mathbf{2 3 . 6 \%} ; \mathrm{A} 1(8 \mathrm{PDs})=$ \\
\hline & & $\mathbf{2 5 . 7 \%} ; \mathrm{B} 1(7 \mathrm{PDs})=\mathbf{3 9 . 2} \%$ \\
\hline \multirow[t]{2}{*}{1245} & Amide III ( $\beta$-sheet, protein) & $\mathrm{A} 3(60 \mathrm{PDs})=\mathbf{2 3 . 8 \%} ; \mathrm{A} 1(8 \mathrm{PDs})=$ \\
\hline & & $\mathbf{4 4 . 2 \%} ; \mathrm{B} 1(7 \mathrm{PDs})=\mathbf{4 5 . 4 \%}$ \\
\hline \multirow[t]{2}{*}{1402} & $\mathrm{C} \beta$ rocking (arginine) & $\mathrm{A} 3(60 \mathrm{PDs})=\mathbf{4 8 . 3 \%} ; \mathrm{A} 1(8 \mathrm{PDs})=$ \\
\hline & & $\mathbf{4 1 . 5 \%} ; \mathrm{B} 1(\mathrm{PDDs})=\mathbf{3 5 . 8} \%$ \\
\hline \multirow[t]{2}{*}{1450} & C-H deformation vibrations & $\mathrm{A} 3(60 \mathrm{PDs})=\mathbf{5 . 6 \%} ; \mathrm{A} 1(8 \mathrm{PDs})=$ \\
\hline & & $\mathbf{3 1 . 0 \%} ; \mathrm{B} 1(7 \mathrm{PDs})=\mathbf{3 2 . 2} \%$ \\
\hline \multirow[t]{2}{*}{1629} & Serine & $\mathrm{A} 3(60 \mathrm{PDs})=\mathbf{4 0 . 2 \%} ; \mathrm{A} 1(8 \mathrm{PDs})=$ \\
\hline & & $\mathbf{4 1 . 1 \%} ; \mathrm{B} 1(7 \mathrm{PDs})=\mathbf{5 1 . 0 \%}$ \\
\hline \multirow[t]{2}{*}{1680} & Amide I, C-O vibrations of & $\mathrm{A} 3(60 \mathrm{PDs})=\mathbf{1 5 . 2 \%} ; \mathrm{A} 1(8 \mathrm{PDs})=$ \\
\hline & polypeptide backbone & $\mathbf{4 0 . 7 \%} ; \mathrm{B} 1(7 \mathrm{PDs})=\mathbf{3 9 . 3 \%}$ \\
\hline
\end{tabular}

